\title{
E-Bayesian estimation based on generalized half Logistic progressive type-II censored data
}

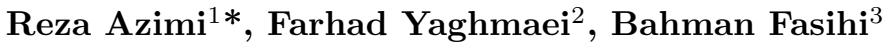 \\ ${ }^{1,3}$ Department of Statistics, Parsabad Moghan Branch, Islamic Azad University, Parsabad Moghan, Iran \\ ${ }^{2}$ Department of Statistics, Faculty of Sciences, Golestan University, Gorgan, Golestan, Iran \\ *Corresponding author E-mail: Azimireza1365@gmail.com
}

\begin{abstract}
In this paper, given a progressively type II censored sample from a generalized half logistic distribution, the Bayesian and E-Bayesian (expectation of the Bayesian estimate) estimators are obtained under LINEX and squared-error loss functions, for the parameter and reliability function. Monte Carlo simulation method is used to generate a progressive Type-II censored data from generalized half logistic distribution, then these data is used to compute the estimations of the parameter and compare both the methods used with different random schemes.
\end{abstract}

Keywords: E-Bayesian estimate, Bayesian estimate, Generalized half logistic distribution, Progressive Type-II censoring.

\section{Introduction}

Let $X=|Z|$, where $Z$ is the standard logistic random variable, $X$ is called the folded or half logistic random variable. The density function of half logistic distribution is a monotonic decreasing function of $x$ is $[0, \infty)$ and has an increasing hazard rate. The generalized versions of half logistic distribution namely Type-I and TypeII were considered along with point estimation of scale parameters and estimation of stress strength reliability based on complete sample by Ramakrishna [2]. Recently Arora et al. [1] considered maximum likelihood estimators of the generalized half logistic distribution under type I progressive censoring with changing failure rates. Azimi et al. [8] obtained Bayes estimators of the parameter and reliability function of generalized half logistic distribution by taking progressive type II censored sample using different loss functions such as LINEX, precautionary and entropy loss functions. The cumulative distribution function (cdf), and probability density function (pdf), of the generalized half logistic distribution with parameter $\beta>0$ are

$$
\begin{aligned}
& F(x \mid \beta)=1-\left[\frac{2 e^{-x}}{1+e^{-x}}\right]^{\beta}, x>0 \\
& f(x \mid \beta)=\frac{\beta\left(2 e^{-x}\right)^{\beta}}{\left(1+e^{-x}\right)^{\beta+1}}
\end{aligned}
$$

The reliability function $R(t)$, at mission time $t$ is given by

$$
R(t)=\left[\frac{2 e^{-t}}{1+e^{-t}}\right]^{\beta},
$$

Progressive Type-II censored sampling is an important method of obtaining data in lifetime studies. A recent account on progressive censoring schemes can be obtained in the monograph by Balakrishnan and Aggarwala [4] or in the excellent review article by Balakrishnan [3]. Suppose that $n$ independent items are put on a test and that 
the lifetime distribution of each item is given by the probability density function of (2). The ordered $m$-failures are observed under the type-II progressively censoring plan $\left(R_{1}, \ldots, R_{m}\right)$ where each $R_{i} \geq 0$ and $\sum_{j=1}^{m} R_{j}+m=n$. If the ordered $m$-failures are denoted by $x_{(1)}<x_{(2)}<\ldots<x_{(m)}$, then the likelihood function based on the observed sample $x_{(1)}<x_{(2)}<\ldots<x_{(m)}$ (for convenience notation are denoted by $x_{1}<x_{2}<\ldots<x_{m}$ ) is

$$
L(\beta)=c \prod_{i=1}^{m} f\left(x_{i} \mid \beta\right)\left[1-F\left(x_{i} \mid \beta\right)\right]^{R_{i}}
$$

where $c=n\left(n-1-R_{1}\right) \ldots\left(n-R_{1}-\ldots-R_{m-1}-m+1\right)$. Substituting (1), (2) in (3), The latter function can be obtained as follows,

$$
\begin{aligned}
L(\beta) & =c \prod_{i=1}^{m} \beta\left(\frac{2 e^{-x_{i}}}{1+e^{-x_{I}}}\right)^{\beta}\left(\frac{2 e^{-x_{i}}}{1+e^{-x_{i}}}\right)^{\beta R_{i}}\left(1+e^{-x_{i}}\right)^{-1} \\
& \propto \beta^{m} \exp \{\beta w\}
\end{aligned}
$$

where

$$
W\left(x_{i}\right)=w=\sum_{i=1}^{m}\left(R_{i}+1\right) \ln \left(\frac{2 e^{-x_{i}}}{1+e^{-x_{i}}}\right) .
$$

\section{Bayesian estimation}

We now derive the Bayes estimator for the parameter and reliability function of the generalized half logistic distribution based on the progressive Type-II censored data. Here we consider family of prior densities as the following form

$$
\pi(\beta)=\frac{b^{a}}{\Gamma(a)} \beta^{a-1} e^{-b \beta}
$$

where $a, b>0$. By combining (4) and the latter prior density function, we can obtain posterior density of $\beta$ as the following form,

$$
\pi(\beta \mid x)=\frac{(b-w)^{m+a}}{\Gamma(m+a)} \beta^{m+a-1} e^{-\beta(b-w)}
$$

Substituting $\beta=\frac{-\log s}{\log \frac{1+e^{-t}}{2 e^{-t}}}$ into (6), we can obtain the posterior density function of $s=R(t)$ as

$$
\pi(s \mid \mathbf{X})=\frac{(b-w)^{m+a}}{\Gamma(m+a)}\left(\log \left\{\frac{1+e^{-t}}{2 e^{-t}}\right\}\right)^{-(m+a)}(-\log s)^{m+a-1} s^{\frac{b-w}{\log \frac{1+e^{-t}}{2 e^{-t}}}}-1
$$

where $0<s<1$.

The Bayesian estimator under the squared-error loss function is given by

$$
\hat{\beta}_{S}=E(\beta \mid \mathbf{X})=\frac{m+a}{b-w}
$$

Under squared-error loss function, the Bayesian estimators of $R(t)$ is given by

$$
\hat{R}_{S}=\left(\frac{b-w}{b-w-\log \frac{2 e^{-t}}{1+e^{-t}}}\right)^{m+a}
$$

Based on LINEX loss function (for more details about the LINEX loss function, see for example, Calabria and Pulicini [9]), we obtain Bayesian estimator of the parameter $\beta$ as the following form (for more details see Azimi et. al [8])

$$
\hat{\beta}_{L}=-\frac{m+a}{k} \log \left(\frac{b-w}{b+k-w}\right)
$$


for estimate $R(t)$ under LINEX loss function we can expand $e^{-k s}$ also in taylor series, and approximate this estimator, the Bayesian estimators under LINEX loss function denoted by $\hat{R}_{L}$ is,

$$
\hat{R}_{L}=-\frac{1}{k} \log \left(1+\sum_{j=1}^{\infty} \frac{(-k)^{j}}{j !}\left(\frac{b-w}{b-w+j T}\right)^{m+a}\right)
$$

where $T=\log \frac{2 e^{-t}}{1+e^{-t}}$.

\section{$3 \quad$ E-Bayesian estimation}

According to Han [5], the prior parameters $a$ and $b$ should be selected to guarantee that $\pi(\beta)$ is a decreasing function of $\beta$. The derivative of $\pi(\beta)$ with respect to $\beta$ is

$$
\frac{d \pi(\beta)}{d \beta}=\frac{b^{a}}{\Gamma(a)} \beta^{a-2} e^{-b \beta}((a-1)-b \beta)
$$

since $a>0, b>0$, and $\beta>0$, it follows $0<a<1, b>0$ due to $\frac{d \pi(\beta)}{d \beta}<0$ and therefore $\pi(\beta)$ is a decreasing function of $\beta$.

Assuming that $a$ and $b$ are independent with bivariate density function

$$
\pi(a, b)=\pi_{1}(a) \pi_{2}(b),
$$

then, the E-Bayesian estimate of $\beta$ (expectation of the Bayesian estimate of $\beta$ ) can be written as

$$
\hat{\beta}_{E B}=E(\beta \mid X)=\iint \hat{\beta}_{B}(a, b) \pi(a, b) d a d b,
$$

where $\hat{\beta}_{B}(a, b)$ is the Bayes estimate of $\beta$ given by (7) and (9). For more details, see Han [6] or jaheen and okasha [7].

\subsection{E-Bayesian estimation under squared-error loss function}

The following distributions of $a$ and $b$ may be used

$$
\begin{array}{ll}
\pi_{1}(a, b)=\frac{2(c-b)}{c^{2}}, & 0<a<1,0<b<c, \\
\pi_{2}(a, b)=\frac{1}{c}, & 0<a<1,0<b<c, \\
\pi_{3}(a, b)=\frac{2 b}{c^{2}}, & 0<a<1,0<b<c,
\end{array}
$$

For $\pi_{1}(a, b)$, the E-Bayesian estimate of $\beta$ is obtained from (7), (11) and (12) as

$$
\begin{aligned}
& \hat{\beta}_{E B S 1}=\iint \hat{\beta}_{S}(a, b) \pi_{1}(a, b) d b d a=\frac{2}{c^{2}} \int_{0}^{1} \int_{0}^{c}\left(\frac{m+a}{b-w\left(x_{i}\right)}\right)(c-b) d b d a \\
& =\frac{2\left(m+\frac{1}{2}\right)}{c^{2}}\left((c-w) \log \left(\frac{c-w}{-w}\right)-c\right)
\end{aligned}
$$

Similarly, the E-Bayesian estimates of $\beta$ based on $\pi_{2}(a, b)$ and $\pi_{3}(a, b)$ are computed and given, respectively, by

$$
\begin{aligned}
& \hat{\beta}_{E B S 2}=\frac{m+\frac{1}{2}}{c} \log \left(\frac{c-w}{-w}\right) \\
& \hat{\beta}_{E B S 3}=\frac{2\left(m+\frac{1}{2}\right)}{c^{2}}\left(c+w \log \left(\frac{c-w}{-w}\right)\right)
\end{aligned}
$$


Under squared-error loss function, the E-Bayesian estimates for the reliability function are computed for the three different distributions of the hyperparameters $a$ and $b$ given by (12). For $\pi_{i}(a, b), i=1,2,3$, the E-Bayesian estimate of the reliability is obtained from (11), (8) and (12) as

$$
\hat{R}_{E B S i}=\iint \hat{R}_{B S} \pi_{i}(a, b) d b d a=\iint\left(\frac{b-w}{b-w-T}\right)^{m+a} \pi_{i}(a, b) d b d a
$$

Since obtaining a closed form expression for $\hat{R}_{E B S i}$ is not possible, We can expand $\hat{R}_{B S}$ also in Taylor series, and approximate $\hat{R}_{E B S i}$. Therefore,

$$
\hat{R}_{B S}=\left(\frac{b-w}{b-w-T}\right)^{m+a}=A_{1}\left[1+a A_{2}+A_{3}+A_{4} a^{2}+A_{5} a b+A_{6} b^{2}\right]
$$

where

$$
A_{1}=\left(\frac{w}{w+T}\right)^{m} \quad, \quad A_{2}=\log \frac{w}{w+T}, \quad A_{3}=-\frac{m T}{(w+T) w}, A_{4}=\frac{1}{2}\left(\log \frac{w}{w+T}\right)^{2}
$$

and

$$
A_{5}=-\frac{m T w \log \frac{w}{w+T}+T w+T^{2}+m T^{2} \log \frac{w}{w+T}}{(w+T)^{2} w}, A_{6}=\frac{1}{2} \frac{-2 m T w+m T^{2}(m-1)}{(w+T)^{2} w^{2}}
$$

Then we can obtain $\hat{R}_{E B S i}$ from (16) and (17) as the following form

$$
\begin{aligned}
& \hat{R}_{E B S 1} \approx A_{1}\left(1+\frac{1}{2} A_{2}+A_{3}+\frac{1}{3} A_{4}+\frac{c}{6} A_{5}+\frac{c^{2}}{6} A_{6}\right) \\
& \hat{R}_{E B S 2} \approx A_{1}\left(1+\frac{1}{2} A_{2}+A_{3}+\frac{1}{3} A_{4}+\frac{c}{4} A_{5}+\frac{c^{2}}{3} A_{6}\right) \\
& \hat{R}_{E B S 3} \approx A_{1}\left(1+\frac{1}{2} A_{2}+A_{3}+\frac{1}{3} A_{4}+\frac{c}{3} A_{5}+\frac{c^{2}}{2} A_{6}\right)
\end{aligned}
$$

\subsection{E-Bayesian estimation under LINEX loss function}

Based on the LINEX loss function, the E-Bayesian estimation of $\beta$ is computed for the three different distributions of the hyperparameters $a$ and $b$ given by (12). For $\pi_{1}(a, b)$, the E-Bayesian estimate of $\beta$ is obtained from (9), (11) and (12) as

$$
\hat{\beta}_{E B L 1}=\frac{m+\frac{1}{2}}{c^{2} k}\left[c^{2} \log G_{1}+\left((k-w)^{2}-2 c(w-k)\right) \log G_{2}+\left(2 c w-w^{2}\right) \log G_{3}-c k\right]
$$

Similarly, the E-Bayesian estimates of $\beta$ based on $\pi_{2}(a, b)$ and $\pi_{3}(a, b)$ are computed and given, respectively, by

$$
\hat{\beta}_{E B L 2}=\frac{m+\frac{1}{2}}{c^{2} k}\left[c^{2} \log G_{1}-c(w-k) \log G_{2}+c w \log G_{3}\right]
$$

and

$$
\hat{\beta}_{E B L 3}=\frac{m+\frac{1}{2}}{c^{2} k}\left[c^{2} \log G_{1}-(k-w)^{2} \log G_{2}+w^{2} \log G_{3}+c k\right]
$$

where

$$
G_{1}=\frac{c-w+k}{c-w}, G_{2}=\frac{c-w+k}{k-w}, \quad G_{3}=\frac{c-w}{-w}
$$

Based on the LINEX loss function, the E-Bayesian estimates for the reliability function are computed for the three different distributions of the hyperparameters $a$ and $b$ given by (12). It follows that, for $\mathrm{i}=1,2,3$, the E-Bayesian estimates of b are obtained from (10), (11) and (12) and written as

$$
\hat{R}_{E B L i}=\iint \hat{R}_{B L} \pi_{i}(a, b) d b d a=\iint-\frac{1}{k} \log \left(1+\sum_{j=1}^{\infty} \frac{(-k)^{j}}{j !}\left(\frac{b-w}{b-w+j T}\right)^{m+a}\right) \pi_{i}(a, b) d b d a
$$

Analytical and numerical computations for the integrals in (21) are very complicated. 


\section{Property of E-Bayesian estimation}

Theorem-1 . For E-Bayesian Estimator of parameter $\beta\left(\hat{\beta}_{E B S i}, i=1,2,3\right)$ when $0<c<w$, we have:

(i) $\hat{\beta}_{E B S 3}<\hat{\beta}_{E B S 2}<\hat{\beta}_{E B S 1}$

(ii) $\lim _{w \rightarrow \infty} \hat{\beta}_{E B S 1}=\lim _{w \rightarrow \infty} \hat{\beta}_{E B S 2}=\lim _{w \rightarrow \infty} \hat{\beta}_{E B S 3}$

Proof (i) From (13),(14) and (15), we have

$$
\hat{\beta}_{E B S 1}-\hat{\beta}_{E B S 2}=\hat{\beta}_{E B S 2}-\hat{\beta}_{E B S 3}=\frac{2\left(m+\frac{1}{2}\right)}{c^{2}}\left[\frac{(c-2 w)}{2} \log \left(1-\frac{c}{w}\right)-c\right]
$$

For $-1<x<1$, we have: $\log (1+x)=x-\frac{x^{2}}{2}+\frac{x^{3}}{3}-\frac{x^{4}}{4}+\frac{x^{5}}{5}+\cdots$

Let $x=\frac{c}{w}$, when $0<c<w, 0<\frac{c}{w}<1$, we get:

$$
\begin{aligned}
{\left[\frac{(c-2 w)}{2} \log \left(1-\frac{c}{w}\right)-c\right] } & =\frac{c-2 w}{2}\left[-\frac{c}{w}-\frac{1}{2} \frac{c^{2}}{w^{2}}-\frac{1}{3} \frac{c^{3}}{w^{3}}-\frac{1}{4} \frac{c^{4}}{w^{4}}-\frac{1}{5} \frac{c^{5}}{w^{5}}-\cdots\right]-c \\
& =c\left[\frac{c^{2}}{w^{2}}\left(\frac{1}{3}-\frac{1}{4}\right)+\frac{c^{3}}{w^{3}}\left(\frac{1}{4}-\frac{1}{6}\right)+\frac{c^{4}}{w^{4}}\left(\frac{1}{5}-\frac{1}{8}\right)+\cdots\right]
\end{aligned}
$$

according to (22) and (23), we have

$$
\hat{\beta}_{E B S 1}-\hat{\beta}_{E B S 2}=\hat{\beta}_{E B S 2}-\hat{\beta}_{E B S 3}>0,
$$

that is

$$
\hat{\beta}_{E B S 3}<\hat{\beta}_{E B S 2}<\hat{\beta}_{E B S 1}
$$

(ii) From (22) and (23) we get

$$
\begin{aligned}
\lim _{w \rightarrow \infty}\left(\hat{\beta}_{E B S 1}-\hat{\beta}_{E B S 2}\right) & =\lim _{w \rightarrow \infty}\left(\hat{\beta}_{E B S 2}-\hat{\beta}_{E B S 3}\right) \\
& =\frac{2\left(m+\frac{1}{2}\right)}{c} \lim _{w \rightarrow \infty}\left[\frac{1}{12} \frac{c^{2}}{w^{2}}+\frac{1}{12} \frac{c^{3}}{w^{3}}+\frac{3}{40} \frac{c^{4}}{w^{4}}+\cdots\right] \\
& =0
\end{aligned}
$$

That is,

$$
\lim _{w \rightarrow \infty} \hat{\beta}_{E B S 1}=\lim _{w \rightarrow \infty} \hat{\beta}_{E B S 2}=\lim _{w \rightarrow \infty} \hat{\beta}_{E B S 3}
$$

Thus, the proof is complete.

Theorem-2. For E-Bayesian Estimator of parameter $\beta$ under Linex loss function $\left(\hat{\beta}_{E B L i}, i=1,2,3\right)$ when $0<c<w$, we have:

(i) $\hat{\beta}_{E B L 1}<\hat{\beta}_{E B L 2}<\hat{\beta}_{E B L 3}$

(ii) $\lim _{w \rightarrow \infty} \hat{\beta}_{E B L 1}=\lim _{w \rightarrow \infty} \hat{\beta}_{E B L 2}=\lim _{w \rightarrow \infty} \hat{\beta}_{E B L 3}$

proof: From (18),(19) and (20), we have

$$
\hat{\beta}_{E B L 1}-\hat{\beta}_{E B L 2}=\hat{\beta}_{E B L 2}-\hat{\beta}_{E B L 3}=\frac{m+\frac{1}{2}}{c^{2} k}\left[\left((k-w)^{2}+c(k-w)\right) \log G_{2}+\left(c w-w^{2}\right) \log G_{3}-c k\right]
$$

according theorem-1 we have

$$
\frac{1}{c^{2}}\left[\left((k-w)^{2}+c(k-w)\right) \log G_{2}+\left(c w-w^{2}\right) \log G_{3}-c k\right]=\frac{(k-w)^{2}+c(k-w)}{c^{2}}
$$




$$
\begin{aligned}
& \times\left[\frac{c}{k-w}-\frac{1}{2} \frac{c^{2}}{(k-w)^{2}}+\frac{1}{3} \frac{c^{3}}{(k-w)^{3}}-\frac{1}{4} \frac{c^{4}}{(k-w)^{4}}+\frac{1}{5} \frac{c^{5}}{(k-w)^{5}}-\cdots\right] \\
& +\frac{c w-w^{2}}{c^{2}}\left[-\frac{c}{w}-\frac{1}{2} \frac{c^{2}}{w^{2}}-\frac{1}{3} \frac{c^{3}}{w^{3}}-\frac{1}{4} \frac{c^{4}}{w^{4}}-\cdots\right] \\
& =\frac{c}{k-w}\left(\frac{1}{3}-\frac{1}{2}\right)+\frac{c^{2}}{(k-w)^{2}}\left(\frac{1}{3}-\frac{1}{4}\right)+\frac{c^{3}}{(k-w)^{3}}\left(\frac{1}{5}-\frac{1}{4}\right)+\cdots \\
& +\frac{c}{w}\left(\frac{1}{3}-\frac{1}{2}\right)+\frac{c^{2}}{w^{2}}\left(\frac{1}{4}-\frac{1}{3}\right)+\frac{c^{3}}{w^{3}}\left(\frac{1}{5}-\frac{1}{4}\right)+\frac{c^{4}}{w^{4}}\left(\frac{1}{6}-\frac{1}{5}\right)+\cdots
\end{aligned}
$$

according to (24) and (25), we have

$$
\hat{\beta}_{E B L 1}-\hat{\beta}_{E B L 2}=\hat{\beta}_{E B L 2}-\hat{\beta}_{E B L 3}<0
$$

that is

$$
\hat{\beta}_{E B L 1}<\hat{\beta}_{E B L 2}<\hat{\beta}_{E B L 3}
$$

(ii) From (24) and (25) we get

$$
\begin{aligned}
\lim _{w \rightarrow \infty}\left(\hat{\beta}_{E B L 1}-\hat{\beta}_{E B L 2}\right) & =\lim _{w \rightarrow \infty}\left(\hat{\beta}_{E B L 2}-\hat{\beta}_{E B L 3}\right) \\
& =\frac{m+\frac{1}{2}}{k} \lim _{w \rightarrow \infty}\left[\frac{c}{k-w}\left(\frac{1}{3}-\frac{1}{2}\right)+\frac{c^{2}}{(k-w)^{2}}\left(\frac{1}{3}-\frac{1}{4}\right)+\frac{c^{3}}{(k-w)^{3}}\left(\frac{1}{5}-\frac{1}{4}\right)+\cdots\right] \\
& +\frac{m+\frac{1}{2}}{k} \lim _{w \rightarrow \infty}\left[\frac{c}{w}\left(\frac{1}{3}-\frac{1}{2}\right)+\frac{c^{2}}{w^{2}}\left(\frac{1}{4}-\frac{1}{3}\right)+\frac{c^{3}}{w^{3}}\left(\frac{1}{5}-\frac{1}{4}\right)+\frac{c^{4}}{w^{4}}\left(\frac{1}{6}-\frac{1}{5}\right)+\cdots\right]
\end{aligned}
$$

That is,

$$
\lim _{w \rightarrow \infty} \hat{\beta}_{E B L 1}=\lim _{w \rightarrow \infty} \hat{\beta}_{E B L 2}=\lim _{w \rightarrow \infty} \hat{\beta}_{E B L 3}
$$

Thus, the proof is complete.

\section{Simulation study}

- We generate $a$ and $b$ from (12)

- For given values of $(a, b)$ we generate $\beta$ from the gamma prior density (5).

Applying the algorithm of Balakrishnan and Aggarwala [4], we used the following steps to generate a progressive Type II censored sample from the Generalized Half Logistic distribution.

1. Simulate $m$ independent exponential random variables $Z_{1}, Z_{2}, \ldots, Z_{m}$.

This can be done using inverse transformation $Z_{i}=-\ln \left(1-U_{i}\right)$ where $U_{i}$ are independent uniform $(0,1)$ random variables.

2. Set

$$
X_{i}=\frac{Z_{1}}{n}+\frac{Z_{2}}{n-R_{1}-1}+\frac{Z_{3}}{n-R_{1}-R_{2}-2}+\cdots+\frac{Z_{i}}{n-R_{1}-R_{2}-\cdots-R_{i-1}-i+1}
$$

for $i=1,2, \ldots, m$. This is the required progressively type-II censored sample from the standard exponential distribution.

3. Finally, we set $Y_{i}=F^{-1}\left(1-\exp \left(-X_{i}\right)\right)$, for $i=1,2, \ldots, m$, where $F^{-1}($.$) is the inverse cumulative distribution$ function of the generalized half logistic distribution. Then $Y_{1}, Y_{2}, \cdots, Y_{m}$ is the required progressively type-II censored sample from the distribution $F($.$) .$ 
4. We compute the the Bayes estimates $\hat{\beta}_{S}, \hat{\beta}_{L}$, respectively using (7), (9), and compute the the E-Bayesian estimates of parameter $\beta$, respectively using, (13), (14), (15), (18), (19), (20).

5. We repeat the above steps 5000 times. We then obtain the means and the MSEs (Mean Squared Error) for different censoring sizes $n, m$ and censoring schemes where

$$
M S E=\frac{1}{5000} \sum_{i=1}^{5000}\left(\phi-\hat{\phi}_{i}\right)^{2}
$$

and $\hat{\phi}$ is the estimator of $\phi$.

Our computational results for the MSE is computed in the above steps, where the values of the parameters used are $a=0.6711976, b=2.539000$ and $c=4$ yielding $\beta=0.3906595$ (as true values). For different progressive censoring scheme $\mathbf{R}$ and various values of $n$ and $m$, the E-Bayesian and Bayesian estimates for the parameters $\beta$ are as in the following Table 1 .

\section{Conclusions}

This paper introduces a new method, called E-Bayesian estimation(see Han [6]), to estimate parameter and reliability function of Generalized Half Logistic distribution when progressive Type II censoring is performed. Based on the results shown in Table-1, one can conclude, Generally, the MSE of the E-Bayesian estimates of $\beta$ are the smallest MSE as the as compared with the Bayesian estimates. The MSE of E-Bayesian estimates under LINEX loss function have smallest MSE as the as compared with the E-Bayesian estimates under squared error loss function. It is immediate to note that MSE of Bayesian and E-Bayesian estimates decrease as $n, m$ increases.

\section{References}

[1] Arora, S. H., Bihmani, G. C., \& Patel, M. N., Some Results on Maximum Likelihood Estimators of Parameters of Generalized Half Logistic Distribution under Type-I Progressive Censoring with Changing Failure Rate, International Journal Of Contemporary Mathematical Sciences 5, 14, (2010), 685-698.

[2] Ramakrsihnan, V., Generalizations to half logistic distribution and related inference, PhD thesis, Acharya Nagarjuna University (AP), India (2008).

[3] Balakrishnan, N., Progressive censoring methodology: an appraisal, TEST 16 (2), (2007), 211-296. http://dx.doi.org/10.1007/s11749-007-0061-y

[4] Balakrishnan, N., Aggarwala, R., Progressive censoring, theory, methods and applications. Birkhauser, Boston, (2000).

[5] Han, M., The structure of hierarchical prior distribution and its applications, Chinese Operation Research and Management Science 6, 3, (1997), 3 1-40.

[6] Han, M., E-Bayesian estimation and hierarchical Bayesian estimation of failure rate, Applied Mathematical Modelling 33, (2009), 1915-1922.

[7] Jaheen, Z. F., Okasha, H. M., E-Bayesian estimation for the Burr type XII model based on type-2 censoring, Applied Mathematical Modelling 35, (2011), 4730-4737.

[8] Azimi, R., yaghmaei, F., \& babanezhad, M., Bayesian Estimation of Generalized Half Logistic Distribution Under Progressive Type-II Censored Data. Applied Mathematical Sciences, 6, 106,(2012), 5253-5261.

[9] Calabria, R., Pulcini, G., Point estimation under asymmetric loss functions for left-truncated exponential samples, Communication in Statistics- Theory and Methods 25, (3), (1996), 585-600. 
Table 1: Averaged values of MSEs for estimates of the parameter $\beta$.

\begin{tabular}{|c|c|c|c|c|c|c|}
\hline$n$ & $m$ & $R=\left(R_{1}, \ldots, R_{m}\right)$ & $\operatorname{MSE}\left(\hat{\beta}_{S}\right)$ & $\operatorname{MSE}\left(\hat{\beta}_{L}\right)(\mathrm{k}=3)$ & $\begin{array}{l}\operatorname{MSE}\left(\hat{\beta}_{E B S 1}\right) \\
\operatorname{MSE}\left(\hat{\beta}_{E B S 2}\right) \\
\operatorname{MSE}\left(\hat{\beta}_{E B S 3}\right)\end{array}$ & $\begin{array}{l}\operatorname{MSE}\left(\hat{\beta}_{E B L 1}\right) \\
\operatorname{MSE}\left(\hat{\beta}_{E B L 2}\right) \\
\operatorname{MSE}\left(\hat{\beta}_{E B L 3}\right)\end{array}$ \\
\hline \multirow[t]{6}{*}{10} & 5 & $(5,0,0,0,0)$ & 0.03585 & 0.01913 & 0.06742 & 0.03131 \\
\hline & & & & & 0.04797 & 0.02361 \\
\hline & & & & & 0.03250 & 0.01775 \\
\hline & 10 & $(0, \ldots, 0)$ & 0.01757 & 0.01306 & 0.02281 & 0.01609 \\
\hline & & & & & 0.01942 & 0.01415 \\
\hline & & & & & 0.01651 & 0.01255 \\
\hline \multirow[t]{6}{*}{20} & 10 & $(5,5,0, \ldots, 0)$ & 0.01711 & 0.01254 & 0.02235 & 0.01557 \\
\hline & & & & & 0.01894 & 0.01361 \\
\hline & & & & & 0.01600 & 0.01199 \\
\hline & 15 & $(5,0, \ldots, 0)$ & 0.01126 & 0.00916 & 0.01329 & 0.01051 \\
\hline & & & & & 0.01192 & 0.00962 \\
\hline & & & & & 0.01072 & 0.00886 \\
\hline \multirow[t]{6}{*}{30} & 20 & $(4,4,2,0, \ldots, 0)$ & 0.00813 & 0.00703 & 0.00912 & 0.00772 \\
\hline & & & & & 0.00844 & 0.00726 \\
\hline & & & & & 0.00784 & 0.00686 \\
\hline & 30 & $(0, \ldots, 0)$ & 0.00540 & 0.00490 & 0.00582 & 0.00521 \\
\hline & & & & & 0.00553 & 0.00500 \\
\hline & & & & & 0.00527 & 0.00482 \\
\hline \multirow[t]{6}{*}{40} & 25 & $(5,5,5,0, \ldots, 0)$ & 0.00640 & 0.00568 & 0.00702 & 0.00613 \\
\hline & & & & & 0.00659 & 0.00583 \\
\hline & & & & & 0.00621 & 0.00556 \\
\hline & 35 & $(1 * 5,0 * 30)$ & 0.00440 & 0.00405 & 0.00468 & 0.00426 \\
\hline & & & & & 0.00448 & 0.00412 \\
\hline & & & & & 0.00430 & 0.00399 \\
\hline \multirow[t]{6}{*}{50} & 40 & $\left(5^{*} 2,0 * 38\right)$ & 0.00414 & 0.00385 & 0.00438 & 0.00403 \\
\hline & & & & & 0.00421 & 0.00391 \\
\hline & & & & & 0.00406 & 0.00380 \\
\hline & 50 & $(0 * 50)$ & 0.00306 & 0.00289 & 0.00319 & 0.00300 \\
\hline & & & & & 0.00310 & 0.00293 \\
\hline & & & & & 0.00302 & 0.00287 \\
\hline
\end{tabular}

